\title{
Dietary patterns during pregnancy and the risk of postpartum depression in Japan: the Osaka Maternal and Child Health Study
}

\author{
Hitomi Okubo ${ }^{1,2_{*}}$, Yoshihiro Miyake ${ }^{3}$, Satoshi Sasaki ${ }^{1,4}$, Keiko Tanaka ${ }^{3}$, Kentaro Murakami ${ }^{4}$, \\ Yoshio Hirota $^{5}$ and the Osaka Maternal and Child Health Study Group \\ ${ }^{1}$ Department of Social and Preventive Epidemiology, Graduate School of Medicine, The University of Tokyo, \\ Hongo 7-3-1, Bunkyo-ku, Tokyo 113-0033, Japan \\ ${ }^{2} J a p a n$ Society for the Promotion of Science, Tokyo, Japan \\ ${ }^{3}$ Department of Public Health, Faculty of Medicine, Fukuoka University, Fukuoka, Japan \\ ${ }^{4}$ Department of Social and Preventive Epidemiology, School of Public Health, The University of Tokyo, Tokyo, Japan \\ ${ }^{5}$ Department of Public Health, Osaka City University Graduate School of Medicine, Osaka, Japan
}

(Received 9 June 2010 - Revised 4 October 2010 - Accepted 19 October 2010 - First published online 13 December 2010)

\section{Abstract}

Although several nutrients and foods are suggested to be preventive against postpartum depression, all previous studies have primarily focused on single nutrients or foods. In contrast, studies on dietary patterns, namely the measurement of overall diet by considering the cumulative effects of nutrient, may provide new insights into the influence of diet on postpartum depression. We prospectively examined the association between dietary patterns during pregnancy and the risk of postpartum depression among 865 Japanese women. Diet was assessed with a validated, self-administered diet history questionnaire. Dietary patterns from thirty-three predefined food groups (energy-adjusted food $(\mathrm{g} / \mathrm{d})$ ) were extracted by factor analysis. Postpartum depression was defined as present when the subjects had an Edinburgh Postnatal Depression Scale score of $\geq 9$ at $2-9$ months postpartum. A total of 121 women (14.0\%) were classified as having postpartum depression. Three dietary patterns were identified: 'Healthy', 'Western' and 'Japanese' patterns. After adjustment for potential confounders, neither the 'Healthy' nor the 'Japanese' pattern was related to the risk of postpartum depression. Compared with the first quartile of the 'Western' pattern, only the second quartile was independently related to a decreased risk of postpartum depression (multivariate OR $0.52,95 \%$ CI $0.30,0.93$ ), although no evident exposure-response associations were observed ( $P$ for trend=0.36). The present study failed to substantiate clear associations between dietary patterns and the risk of postpartum depression. Further studies with more accurate measurements are warranted to confirm the relationship between dietary patterns and the risk of postpartum depression.

Key words: Cohort studies: Dietary patterns: Postpartum depression: Japanese pregnant women

Postpartum depression, a mental and emotional condition that emerges within several weeks after delivery, can exert detrimental effects on maternal, infant and family well-being $^{(1)}$. Investigation of risk factors for and the treatment of postpartum depression is thus warranted in community health.

As one modifiable risk factor, nutrition has been suggested to play a role in postpartum depression ${ }^{(2,3)}$. Epidemiological studies have focused primarily on $\mathrm{B}$ vitamins (folate, vitamins $\mathrm{B}_{2}, \mathrm{~B}_{6}$ and $\left.\mathrm{B}_{12}\right)^{(3-5)}$ and longchain $n-3$ PUFA $^{(6-9)}$ on the basis of their role in the biosynthesis, metabolism and function of various hormones and neurotransmitters, but results have not been consistent. One reason for this inconsistency may be the traditional single nutrient- or food-based approach. This dominant approach examines single nutrients or foods but might not adequately account for complicated interactions and cumulative effects and might result in the drawing of erroneous associations between dietary factors and disease.

Recently, the dietary pattern approach, which measures overall diet, has been widely used to elucidate the relationship between diet and several health outcomes ${ }^{(10,11)}$. Results have suggested the usefulness of this analysis in capturing some of the complexity of diet ${ }^{(12)}$. To our knowledge, however, the relationship between dietary

Abbreviations: DHQ, dietary history questionnaire; OMCHS, Osaka Maternal and Child Health Study.

*Corresponding author: H. Okubo, fax +81 35841 7873, email okubo@m.u-tokyo.ac.jp

† Other members of the study group are listed in the Appendix. 
patterns and the risk of postpartum depression has not been investigated.

In the present study, we examined the association between dietary patterns during pregnancy and the risk of postpartum depression among 865 Japanese women using data from the Osaka Maternal and Child Health Study (OMCHS). We hypothesised that a dietary pattern high in the main food sources of nutrients, such as $\mathrm{B}$ vitamins including folate (from vegetables and fruits) and $n$-3 PUFA (from fish and shellfish), potentially protective against postpartum-depressive symptoms would be associated with a decreased risk of postpartum depression.

\section{Subjects and methods}

\section{Study population}

Subjects were pregnant women who participated in the OMCHS, a prospective cohort study investigating the preventive and risk factors for maternal and child health problems. The principal objective of the OMCHS is to clarify risk factors for childhood allergic disorders. Details of the OMCHS have been published elsewhere ${ }^{(5,7)}$. Briefly, a total of 1002 pregnant women in Osaka Prefecture took part in a baseline survey conducted between November 2001 and March 2003, of whom 867 participated in the second survey at 2-9 months postpartum. After excluding two women with missing information on baby's birth weight, the final analysis comprised 865 women. The present study was conducted according to the guidelines laid down in the Declaration of Helsinki, and all procedures involving human subjects/patients were approved by the ethics committee of the Osaka City University School of Medicine. Written informed consent was obtained from all subjects/patients.

\section{Measurements}

At the baseline survey, each participant filled out a set of two self-administered questionnaires. A third selfadministered questionnaire was additionally answered in the second survey. Participants mailed these completed questionnaires to the data management centre at the time of each survey. Research technicians completed missing or illogical data by telephone interview.

In the baseline survey, we collected information on dietary habits during the preceding month using a validated, self-administered, comprehensive, dietary history questionnaire $(\mathrm{DHQ})^{(13,14)}$. Estimates of daily intake for 150 food and beverage items, energy and nutrients were calculated using an ad boc computer algorithm for the DHQ, which was based on the Standard Tables of Food Composition in Japan ${ }^{(15,16)}$. According to similar nutrient profiles and culinary usage of the food items, 145 food items of the DHQ, excluding five items (cornflakes, nutritional supplement bars, Japanesestyle pancakes, noodle soup and drinking-water), were grouped into thirty-three predefined food groups by adding food items within each group to extract dietary patterns ${ }^{(17)}$. Information on dietary supplements was not used in the calculation of dietary intake because of a lack of a reliable composition table in Japan. A more detailed description of the methods used to calculate dietary intake and the validity of the DHQ has been published elsewhere ${ }^{(13,14)}$.

Another self-administered questionnaire at the baseline survey inquired about age, gestation, parity, smoking habits, family structure, occupation, family income, education, weight, height and changes in diet in the previous 1 month. BMI was calculated by dividing self-reported body weight $(\mathrm{kg})$ by the square of self-reported height $\left(\mathrm{m}^{2}\right)$.

The self-administered questionnaire in the second survey included the Japanese version ${ }^{(18)}$ of the Edinburgh Postnatal Depression Scale ${ }^{(19,20)}$, a ten-item self-reported scale designed to screen for postpartum depression in community samples. Each item is scored on a four-point scale (from 0 to 3), giving a total score range of $0-30$. The scale rates the intensity of depressive symptoms present within the preceding $7 \mathrm{~d}$. Although a cut-off of $9 / 10$ has been proposed to identify postpartum-depressive symptoms ${ }^{(19)}$, a cut-off of $8 / 9$ was found to detect depression among Japanese women with a specificity of $93 \%$ and a sensitivity of $75 \%{ }^{(18)}$. Postpartum depression in the present study was therefore defined as present when the Edinburgh Postnatal Depression Scale score was $\geq 9$. The questionnaire also elicited information on medical problems in pregnancy as well as on the sex, birth weight and date of birth of the infant born after the baseline survey.

\section{Statistical analysis}

All statistical analyses were performed using Statistical Analysis Systems statistical software version 9.1 (SAS Institute, Inc., Cary, NC, USA).

Factor analysis to derive dietary patterns based on the thirty-three predefined food groups from the DHQ was conducted using the FACTOR PROCEDURE of the SAS software. Intakes of these food groups ( $\mathrm{g}$ food/d) were adjusted for total energy intake using the residual $\operatorname{method}^{(21)}$. The number of factors was determined by scree plots and the combination of foods on the factors. A factor solution with three factors was found to be reasonable and meaningful. The proportion of variance explained by each factor was calculated by dividing the sum of the squares of the respective factor loadings by the number of variables. The factor score for each dietary pattern was calculated by summing the intakes of food groups weighted by their factor loadings ${ }^{(22)}$, with each subject receiving a factor score for each identified pattern. We used these scores to rank the subjects according to the 
degree to which they conformed to the dietary pattern. All data presented in the present study are from the Varimax rotation. The validity of dietary patterns derived from the DHQ has been reported previously ${ }^{(17)}$.

Pearson's correlation coefficients were calculated between each score for dietary pattern and energyadjusted nutrients such as $\mathrm{B}$ vitamins (vitamins $\mathrm{B}_{2}, \mathrm{~B}_{6}$, $\mathrm{B}_{12}$ and folate) and $n-3$ PUFA (total $n-3$ PUFA, EPA and DHA). To compare the risk of postpartum depression, scores for each dietary pattern were categorised into quartiles based on the distribution of the study participants. The first quartile of each dietary pattern was used as a reference category. Logistic regression analysis was used to estimate both crude and multivariate ratios (OR) and 95\% CI of postpartum depression (the dependent variable) for each quartile category of dietary pattern (independent variables). Multivariate OR were calculated by adjusting for potential confounding factors ${ }^{(5,7)}$, including age (years, continuous), gestation (weeks, continuous), parity ( 0 and $\geq 1$ ), cigarette smoking (never, former and current), change in diet in the preceding 1 month (none or seldom, slight and substantial), family structure (nuclear and expanded), occupation (outside work and housewife), family income $(<4000000,4000000-5999999$ and $\geq 6000000 ¥$ year), education $(<13,13-14$ and $\geq 15$ years), season when data at the baseline survey were collected (spring, summer, autumn and winter), BMI ( $\mathrm{kg} / \mathrm{m}^{2}$, continuous), time of delivery before the second survey ( $<4$ and $\geq 4$ months), medical problems in pregnancy (yes and no), baby's sex (male and female) and baby's birth weight (g, continuous). Trends of association were assessed by a logistic regression model assigning consecutive integers $(1-4)$ to the levels of the independent variables. Possible interactions between dietary patterns and several characteristics were also examined in the multivariate model. All reported $P$ values were two-tailed, and a $P$ value of $<0.05$ was considered to be statistically significant

\section{Results}

The subjects' characteristics are listed in Table 1. Of the 865 women, $591(68.4 \%)$ took part in the baseline survey by the 20th week of gestation. A total of 121 subjects $(14.0 \%)$ were classified as having postpartum depression.

Three dietary patterns were identified. The factorloading matrices for each dietary pattern are shown in Table 2. Factor 1, which loaded heavily on green and yellow vegetables, seaweeds, white vegetables, potatoes, fish, fruits, shellfish and sea products, was labelled the 'Healthy' pattern. Factor 2, with high loadings for vegetable oil, beef and pork, salt-containing seasonings, processed meat, chicken and eggs, was labelled the 'Western' pattern. Factor 3, with high loadings for rice, miso soup, sea products, fish and pickled vegetables, was labelled the 'Japanese' pattern. Overall, the three dietary patterns accounted for $22 \cdot 1 \%$ of the overall variance in food intake. The Pearson correlation coefficients between each dietary pattern and nutrients, such as $\mathrm{B}$ vitamins (vitamins $\mathrm{B}_{2}, \mathrm{~B}_{6}, \mathrm{~B}_{12}$ and folate) and $n-3$ PUFA (total $n-3$ PUFA, EPA and DHA), potentially protective against postpartumdepressive symptoms were examined. The 'Healthy' pattern was significantly correlated with the intake of vitamin $\mathrm{B}_{2}\left(\begin{array}{r}0.42\end{array}\right)$, vitamin $\mathrm{B}_{6}\left(\begin{array}{lll}r & 0.71\end{array}\right)$, vitamin $\mathrm{B}_{12}$ ( $r$ 0.48), folate $(r$ 0.69), total $n$-3 PUFA $(r$ 0.26), DHA $(r 0.40)$ and EPA $(r 0.42)$. The 'Western' pattern was significantly correlated with the intake of total $n$-3 PUFA $(r 0.51)$. In contrast, the 'Japanese' pattern was significantly correlated with the intake of total $n$-3 PUFA ( $r$ 0.33), DHA $(r 0.46)$ and EPA $(r$ 0.48).

Crude and multivariate OR (95\% CI) for postpartum depression across quartiles of all three dietary patterns are shown in Table 3. No association was observed

Table 1. Characteristics of 865 Japanese women who participated in the Osaka Maternal and Child Health Study, Japan

(Mean values and standard deviations or percentages)

\begin{tabular}{|c|c|c|}
\hline \multirow[b]{2}{*}{ Variable } & \multicolumn{2}{|c|}{ All $(n 865)$} \\
\hline & Mean & SD \\
\hline \multicolumn{3}{|l|}{ Baseline characteristics } \\
\hline Age (years) & $29 \cdot 9$ & $4 \cdot 0$ \\
\hline BMI $\left(\mathrm{kg} / \mathrm{m}^{2}\right)$ & $21 \cdot 5$ & $2 \cdot 8$ \\
\hline Gestation (weeks) & $18 \cdot 0$ & $6 \cdot 8$ \\
\hline Parity of 1 or more (\%) & \multicolumn{2}{|c|}{50.9} \\
\hline \multicolumn{3}{|l|}{ Cigarette smoking (\%) } \\
\hline Never & \multicolumn{2}{|c|}{$71 \cdot 6$} \\
\hline Former & \multicolumn{2}{|c|}{$11 \cdot 8$} \\
\hline Current & \multicolumn{2}{|c|}{$16 \cdot 7$} \\
\hline \multicolumn{3}{|c|}{ Changes in diet in the previous 1 month (\%) } \\
\hline None or seldom & \multicolumn{2}{|c|}{$28 \cdot 8$} \\
\hline Slight & \multicolumn{2}{|c|}{$44 \cdot 3$} \\
\hline Substantial & \multicolumn{2}{|c|}{$26 \cdot 9$} \\
\hline Nuclear family structure (\%) & \multicolumn{2}{|c|}{$87 \cdot 3$} \\
\hline \multicolumn{3}{|l|}{ Occupation (\%) } \\
\hline Outside work & \multicolumn{2}{|c|}{$29 \cdot 0$} \\
\hline Housewife & \multicolumn{2}{|c|}{$71 \cdot 0$} \\
\hline \multicolumn{3}{|l|}{ Family income (¥/year, \%) } \\
\hline$<4000000$ & \multicolumn{2}{|c|}{$28 \cdot 9$} \\
\hline $4000000-5999999$ & \multicolumn{2}{|c|}{39.9} \\
\hline$\geq 6000000$ & \multicolumn{2}{|c|}{$31 \cdot 2$} \\
\hline \multicolumn{3}{|l|}{ Education (years, \%) } \\
\hline$<13$ & \multicolumn{2}{|c|}{$29 \cdot 7$} \\
\hline $13-14$ & \multicolumn{2}{|c|}{$42 \cdot 4$} \\
\hline$\geq 15$ & \multicolumn{2}{|c|}{$27 \cdot 9$} \\
\hline \multicolumn{3}{|l|}{ Season when data were collected (\%) } \\
\hline Spring & \multicolumn{2}{|c|}{$32 \cdot 1$} \\
\hline Summer & \multicolumn{2}{|c|}{$16 \cdot 4$} \\
\hline Autumn & \multicolumn{2}{|c|}{$22 \cdot 0$} \\
\hline Winter & & \\
\hline Characteristics at the postnatal assessn & & \\
\hline Postpartum depression (\%)* & & \\
\hline Time of delivery before the assessme & hs, \%) & \\
\hline$<4$ & & \\
\hline$\geq 4$ & & \\
\hline Medical problems in pregnancy $(\%) \dagger$ & & \\
\hline Baby's sex (male, \%) & & \\
\hline Baby's birth wt (g) & 3069 & 414 \\
\hline
\end{tabular}

* Defined according to the Edinburgh Postnatal Depression Scale score of $\geq 9^{(18)}$. $\dagger$ Hyperemesis, hydramnios, oligoamnios, gestosis, abruptio placenta, placenta previa or incompetent cervical os. 
Table 2. Factor-loading matrix for the major dietary patterns identified from a self-administered diet history questionnaire among 865 Japanese women who participated in the Osaka Maternal and Child Health Study, Japan

\begin{tabular}{|c|c|c|c|}
\hline & $\begin{array}{l}\text { Factor } 1 \\
\text { (Healthy) }\end{array}$ & $\begin{array}{c}\text { Factor } 2 \\
\text { (Western) }\end{array}$ & $\begin{array}{c}\text { Factor } 3 \\
\text { (Japanese) }\end{array}$ \\
\hline \multicolumn{4}{|l|}{ Food group } \\
\hline Green and yellow vegetables & $0 \cdot 70^{\star}$ & $0 \cdot 15$ & 0.00 \\
\hline Seaweeds & $0.62^{*}$ & 0.01 & $0 \cdot 15$ \\
\hline White vegetables & $0.61^{*}$ & $0.33^{*}$ & 0.01 \\
\hline Mushrooms & $0.61^{*}$ & 0.03 & 0.07 \\
\hline Pulses & $0.58^{\star}$ & 0.09 & $0 \cdot 19$ \\
\hline Potatoes & $0.46^{*}$ & -0.02 & 0.12 \\
\hline Fish & $0.39^{*}$ & -0.05 & $0.36^{\star}$ \\
\hline Fruits & $0.37^{*}$ & $-0.33^{\star}$ & -0.05 \\
\hline Shellfish & $0.27^{\star}$ & -0.01 & 0.21 \\
\hline Sugar & $0 \cdot 10$ & 0.06 & 0.01 \\
\hline Alcoholic beverages & -0.11 & $0 \cdot 11$ & 0.05 \\
\hline Noodles & -0.17 & -0.07 & 0.12 \\
\hline Soft drinks & $-0.34^{\star}$ & $-0.29^{\star}$ & 0.02 \\
\hline Vegetable oil & -0.10 & $0.57^{*}$ & 0.03 \\
\hline Beef and pork & 0.03 & $0.55^{\star}$ & 0.05 \\
\hline Salt-containing seasonings & 0.21 & $0.53^{*}$ & 0.11 \\
\hline Processed meat & -0.08 & $0.42^{\star}$ & -0.01 \\
\hline Chicken & 0.15 & $0.42^{\star}$ & 0.07 \\
\hline Eggs & 0.04 & $0.39^{*}$ & 0.03 \\
\hline Butter & 0.04 & 0.21 & -0.12 \\
\hline Japanese and Chinese tea & 0.03 & $0 \cdot 15$ & $0 \cdot 12$ \\
\hline Tea & -0.04 & -0.06 & -0.01 \\
\hline Nuts & 0.20 & -0.21 & -0.13 \\
\hline Fruit and vegetable juice & -0.04 & -0.24 & 0.03 \\
\hline Rice & -0.12 & -0.05 & $0.68^{*}$ \\
\hline Miso soup & 0.12 & $-0 \cdot 10$ & $0.55^{\star}$ \\
\hline Sea products & $0.32^{*}$ & 0.02 & $0.42^{\star}$ \\
\hline Pickled vegetables & 0.13 & 0.03 & $0 \cdot 27^{\star}$ \\
\hline Other soup & 0.02 & 0.07 & 0.11 \\
\hline Coffee and cocoa & 0.00 & $0 \cdot 10$ & $-0 \cdot 11$ \\
\hline Dairy products & 0.23 & -0.06 & $-0.32^{\star}$ \\
\hline Confectioneries & $-0.28^{*}$ & $-0.34^{\star}$ & $-0.45^{\star}$ \\
\hline Bread & -0.06 & -0.15 & $-0.65^{\star}$ \\
\hline Variation explained (\%) & 9.4 & $6 \cdot 4$ & $6 \cdot 3$ \\
\hline
\end{tabular}

${ }^{*}$ Absolute values $<-0.25$ or $>0.25$.

between the 'Healthy' pattern and the risk of postpartum depression. Compared with the first quartile of the 'Western' pattern, only the second quartile of this pattern was significantly associated with a decreased risk of postpartum depression. This association remained significant after adjustment for possible confounding factors, albeit with slight attenuation (multivariate OR 0.52 , 95\% CI $0.30,0.93)$. Compared with the first quartile of the 'Japanese' pattern, only the second quartile of this pattern was associated with a lower risk of postpartum depression, but the association disappeared after adjustment for possible confounding factors (multivariate $\mathrm{OR}$ $0.56,95 \%$ CI $0.30,1 \cdot 02$ ).

\section{Discussion}

The primary aim of the present study was to investigate the relationship between dietary patterns during pregnancy and the risk of postpartum depression. We hypothesised that a dietary pattern high in the main food sources of nutrients, such as B vitamins including folate (from vegetables and fruits) and marine-originated $n$-3 PUFA (from fish and shellfish), potentially protective against postpartum-depressive symptoms would be related to a decreased risk of postpartum depression ${ }^{(23,24)}$. In the present study, we found the fact that the 'Healthy' pattern, characterised by a high intake of green and yellow vegetables, seaweeds, white vegetables, potatoes, fish, fruits, shellfish and sea products, was significantly correlated with the intake of vitamins $\mathrm{B}_{2}, \mathrm{~B}_{6}, \mathrm{~B}_{12}$, folate, total n-3 PUFA, DHA and EPA. However, no evident association was observed between the 'Healthy' pattern and the risk of postpartum depression in the present study. Furthermore, our previous examination of the relationship between postpartum depression and dietary intake at the food ${ }^{(7)}$ and nutrient levels ${ }^{(5)}$ among the same subjects also showed no clear inverse relationships between the intake of fish, $n-3$ PUFA, folate and B vitamins and the risk of postpartum depression $^{(5,7)}$. In the present study of Japanese women, we could not substantiate evident exposure-response associations between dietary patterns and the risk of postpartum depression regardless of the consideration of the cumulative effects of possibly protective nutrients, although only the second quartile of the 'Western' pattern was associated with the lower risk of postpartum depression.

To our knowledge, this is the first study to examine the relationship between dietary patterns and the risk of postpartum depression. To date, however, only three studies have examined the relationship of dietary pattern with depression among general populations ${ }^{(25-27)}$. In a large British middle-aged population ${ }^{(25)}$, a 'whole food' pattern (vegetables, fruits and fish) was associated with a lower risk of 5 years latter depressive symptoms, whereas a 'processed food' pattern (sweetened desserts, fried food, processed meat, refined grains and high-fat dairy products) was associated with a higher risk. Similar findings were also observed in the Traditional dietary pattern and the Western pattern among Australian women $^{(26)}$. In a study of participants in the SUN Project ${ }^{(27)}$, higher adherence to the Mediterranean dietary pattern, characterised by high intakes of vegetables, fruit and nuts, cereal, legumes and fish, a high ratio of MUFA:SFA, moderate alcohol, a low intake of meat products and whole-fat dairy products, was associated with a lower risk of the incidence of clinical depression. The present findings are at variance with these previous studies. The reason for this inconsistency is unknown, but pathophysiological differences such as the drastic change in hormonal level throughout pregnancy and increasing overall nutrient requirements of pregnant women might be related ${ }^{(3,28)}$.

Several limitations of the present study warrant mention. First, the diagnosis of postpartum depression was established by a self-report rating scale (i.e. Edinburgh Postnatal Depression Scale) rather than by 
Table 3. Crude and multivariate odds ratios $(95 \% \mathrm{Cl})$ for postpartum depression according to the quartile of dietary pattern scores among 865 Japanese women who participated in the Osaka Maternal and Child Health Study, Japan

(Odds ratios and $95 \%$ confidence intervals)

\begin{tabular}{|c|c|c|c|c|c|c|c|c|c|}
\hline \multirow[b]{3}{*}{ Variable } & \multicolumn{8}{|c|}{ Quartile of dietary pattern } & \multirow[b]{3}{*}{$P$ for trend } \\
\hline & \multirow{2}{*}{\multicolumn{2}{|c|}{$\frac{1(n 216)}{\text { OR }}$}} & \multicolumn{2}{|r|}{$2(n 216)$} & \multicolumn{2}{|c|}{$3(n 217)$} & \multicolumn{2}{|c|}{$4(n 216)$} & \\
\hline & & & OR & $95 \% \mathrm{Cl}$ & OR & $95 \% \mathrm{Cl}$ & OR & $95 \% \mathrm{Cl}$ & \\
\hline \multicolumn{10}{|l|}{ Factor 1 (Healthy) } \\
\hline$n$ with postpartum depression & & 30 & & 25 & & & & & \\
\hline Crude & 1.00 & & 0.81 & $0.46,1.43$ & $1 \cdot 32$ & $0.78,2 \cdot 22$ & 0.92 & $0.53,1.61$ & $0 \cdot 76$ \\
\hline Multivariate $^{*}$ & 1.00 & & 0.82 & $0.46,1.47$ & 1.49 & $0.86,2.60$ & 0.94 & $0.52,1.69$ & 0.72 \\
\hline \multicolumn{10}{|l|}{ Factor 2 (Western) } \\
\hline$n$ with postpartum depression & & 40 & & 22 & & & & & \\
\hline Crude & 1.00 & & 0.50 & $0.29,0.87$ & 0.68 & $0.40,1 \cdot 14$ & 0.71 & $0.42,1.19$ & 0.31 \\
\hline Multivariate $^{\star}$ & 1.00 & & 0.52 & $0.30,0.93$ & 0.71 & $0.41,1.20$ & 0.73 & $0.42,1.24$ & 0.36 \\
\hline \multicolumn{10}{|l|}{ Factor 3 (Japanese) } \\
\hline$n$ with postpartum depression & & 34 & & 20 & & & & & \\
\hline Crude & 1.00 & & 0.55 & $0.30,0.98$ & 1.03 & $0.62,1.72$ & 0.93 & $0.55,1.57$ & 0.70 \\
\hline Multivariate* & 1.00 & & 0.56 & $0.30,1.02$ & $1 \cdot 14$ & $0.66,1.96$ & 0.96 & $0.56,1.64$ & 0.59 \\
\hline
\end{tabular}

a clinician-administered structured diagnostic interview. The Edinburgh Postnatal Depression Scale was originally designed as a screening test for depressive symptoms within the previous $7 \mathrm{~d}$. Moreover, the second survey for the assessment of postpartum-depressive symptoms was conducted at 2-9 months postpartum. However, almost all subjects (89.2\%) took part in the second survey at 3-4 months postpartum. Therefore, accurate estimation of the incidence and prevalence of postpartum depression might not be obtained due to the limited assessment period.

Second, dietary data were obtained using a selfadministered diet history questionnaire. Although the validity and reliability of our dietary questionnaire have been evaluated ${ }^{(13,14,17)}$, non-differential misclassification might also exist in dietary exposures, and this may bias the magnitude of the observed effects towards null. Moreover, our dietary questionnaire was designed to assess the recent diet, namely the preceding 1 month before completing the questionnaire, rather than throughout pregnancy. However, previous longitudinal studies have reported that dietary patterns do not vary significantly throughout pregnancy ${ }^{(29,30)}$. We also assessed a change in diet during the assessment period to account for the possibility that the diet was changed due to reasons such as hyperemesis gravidarum. We found no significant interaction between changes in diet in the past month and the dietary patterns under investigation in relation to postpartum depression (data not shown). Potential misclassification associated with variability across subjects with regard to the stability of diet during pregnancy is thus likely to be negligible. The negative findings of the present study are therefore unlikely to be ascribable to dietary assessment during pregnancy.
Third, because of a lack of information on taking antidepressant medication, we could not control for taking anti-depressant medication. In addition, although we attempted to adjust for a number of potential confounding variables, unknown or poorly measured confounders could not be controlled. In particular, we could not control for personal and family psychiatric history, sociocultural factors or personal and family relationships because of a lack of information.

Fourth, the study subjects were not a representative sample of Japanese women in the general population, and the present findings might not be generalised, although the relatively high follow-up rate (86.3\%) minimised the possibility of bias by loss of follow-up. Finally, because our sample size was relatively small, statistical power may have been insufficient to allow detection of the association between dietary patterns and postpartum depression.

In conclusion, this prospective study in Japanese women failed to substantiate clear exposure-response relationships of dietary patterns with the subsequent risk of postpartum depression, although the second quartile of the 'Western' pattern was associated with a lower risk of postpartum depression. Further large-scale prospective studies are needed to confirm the relationship between dietary patterns and the risk of postpartum depression, given the paucity of evidence on this issue.

\section{Acknowledgements}

We thank the Neyagawa City Government, Hirakata City Government, Katano City Government, Shijonawate City Government, Kaizuka City Government, Takaishi City Government, Hannan City Government, Neyagawa 
City Medical Association, Hirakata City Medical Association and Kadoma City Medical Association for their valuable support. The present study was supported in part by a Grant-in-Aid (13770206, 16790351) for Scientific Research from the Ministry of Education, Culture, Sports, Science and Technology and Health and Labour Sciences Research Grants, Research on Allergic Disease and Immunology from the Ministry of Health, Labour, and Welfare, and a Grant-in-Aid for Japan Society for the Promotion of Science Fellows (21-3370) from the Japan Society for the Promotion of Science. H. O. conducted the statistical analyses and wrote the manuscript. Y. M. and S. S. contributed to the planning of the OMCHS and data collection and assisted in manuscript preparation. K. T. contributed to data collection. K. M. assisted in the manuscript preparation. Y. H. supervised the design and execution of the OMCHS. All authors contributed to and approved the final manuscript. None of the authors had any personal or financial conflict of interest to declare.

\section{References}

1. Horowitz JA \& Goodman JH (2005) Identifying and treating postpartum depression. J Obstet Gynecol Neonatal Nurs 34, 264-273.

2. Bodnar LM \& Wisner KL (2005) Nutrition and depression: implications for improving mental health among childbearing-aged women. Biol Psychiatry 58, 679-685.

3. Leung BM \& Kaplan BJ (2009) Perinatal depression: prevalence, risks, and the nutrition link - a review of the literature. J Am Diet Assoc 109, 1566-1575.

4. Livingston JE, MacLeod PM \& Applegarth DA (1978) Vitamin $\mathrm{B}_{6}$ status in women with postpartum depression. Am J Clin Nutr 31, 886-891.

5. Miyake Y, Sasaki S, Tanaka K, et al. (2006) Dietary folate and vitamins $\mathrm{B}_{12}, \mathrm{~B}_{6}$, and $\mathrm{B}_{2}$ intake and the risk of postpartum depression in Japan: the Osaka Maternal and Child Health Study. J Affect Disord 96, 133-138.

6. Doornbos B, van Goor SA, Dijck-Brouwer DA, et al. (2009) Supplementation of a low dose of DHA or DHA + AA does not prevent peripartum depressive symptoms in a small population based sample. Prog Neuropsychopharmacol Biol Psychiatry 33, 49-52.

7. Miyake Y, Sasaki S, Yokoyama T, et al. (2006) Risk of postpartum depression in relation to dietary fish and fat intake in Japan: the Osaka Maternal and Child Health Study. Psychol Med 36, 1727-1735.

8. Otto SJ, de Groot RH \& Hornstra G (2003) Increased risk of postpartum depressive symptoms is associated with slower normalization after pregnancy of the functional docosahexaenoic acid status. Prostaglandins Leukot Essent Fatty Acids 69, 237-243.

9. Strom M, Mortensen EL, Halldorsson TI, et al. (2009) Fish and long-chain $n-3$ polyunsaturated fatty acid intakes during pregnancy and risk of postpartum depression: a prospective study based on a large national birth cohort. Am J Clin Nutr 90, 149-155.

10. Kant AK (2004) Dietary patterns and health outcomes. $J$ Am Diet Assoc 104, 615-635.

11. Newby PK \& Tucker KL (2004) Empirically derived eating patterns using factor or cluster analysis: a review. Nutr Rev 62, $177-203$.
12. Moeller SM, Reedy J, Millen AE, et al. (2007) Dietary patterns: challenges and opportunities in dietary patterns research an Experimental Biology workshop, April 1, 2006. J Am Diet Assoc 107, 1233-1239.

13. Sasaki S, Ushio F, Amano K, et al. (2000) Serum biomarkerbased validation of a self-administered diet history questionnaire for Japanese subjects. J Nutr Sci Vitaminol 46, 285-296.

14. Sasaki S, Yanagibori R \& Amano K (1998) Selfadministered diet history questionnaire developed for health education: a relative validation of the test-version by comparison with 3-day diet record in women. $J$ Epidemiol 8, 203-215.

15. Science and Technology Agency (2005) Standard Tables of Food Composition in Japan (in Japanese), 5th rev ed. Tokyo: Printing Bureau of the Ministry of Finance.

16. Science and Technology Agency (2005) Standard Tables of Food Composition in Japan: Fatty Acid Section (in Japanese), 5th rev and enlarged ed. Tokyo: Printing Bureau of the Ministry of Finance.

17. Okubo H, Murakami K, Sasaki S, et al. (2010) Relative validity of dietary patterns derived from a self-administered diet history questionnaire using factor analysis among Japanese adults. Public Health Nutr 13, 1080-1089.

18. Okano T, Murata M, Masuji F, et al. (1996) Validation and reliability of Japanese version of the EPDS. Arch Psychiatr Diag Clin Eval 7, 525-533 (in Japanese).

19. Cox J (1987) Origins and development of the 10 item Edinburgh Depression Scale. In Perinatal Psychiatry: Use and Misuse of the Edinburgh Postnatal Depression Scale, pp. 115-124 [J Cox and J Holden, editors]. London: Gaskell.

20. Cox JL, Holden JM \& Sagovsky R (1987) Detection of postnatal depression. Development of the 10-item Edinburgh Postnatal Depression Scale. Br J Psychiatry 150, 782-786.

21. Willett W \& Stampfer MJ (1986) Total energy intake: implications for epidemiologic analyses. Am J Epidemiol 124, $17-27$

22. Kim JO \& Mueller CW (1978) Factor Analysis: Statistical Methods and Practical Issues. Thousand Oaks, CA: Sage Publications, Inc.

23. Hibbeln JR (1998) Fish consumption and major depression. Lancet 351, 1213.

24. Hibbeln JR (2002) Seafood consumption, the DHA content of mothers' milk and prevalence rates of postpartum depression: a cross-national, ecological analysis. J Affect Disord 69, 15-29.

25. Akbaraly TN, Brunner EJ, Ferrie JE, et al. (2009) Dietary pattern and depressive symptoms in middle age. $\mathrm{Br} J$ Psychiatry 195, 408-413.

26. Jacka FN, Pasco JA, Mykletun A, et al. (2010) Association of Western and traditional diets with depression and anxiety in women. Am J Psychiatry 167, 305-311.

27. Sanchez-Villegas A, Bes-Rastrollo M, Martinez-Gonzalez MA, et al. (2006) Adherence to a Mediterranean dietary pattern and weight gain in a follow-up study: the SUN cohort. Int J Obes (Lond) 30, 350-358.

28. Jenkins DJA, Wolever TMS, Taylor RH, et al. (2003) Endocrine factors and postpartum depression. A selected review. J Reprod Med 48, 402-408.

29. Crozier SR, Robinson SM, Godfrey KM, et al. (2009) Women's dietary patterns change little from before to during pregnancy. J Nutr 139, 1956-1963.

30. Cuco G, Fernandez-Ballart J, Sala J, et al. (2006) Dietary patterns and associated lifestyles in preconception, pregnancy and postpartum. Eur J Clin Nutr 60, 364-371. 


\section{Appendix}

Space limitations preclude the inclusion as authors of the following members of the OMCHS Group: Hideharu Kanzaki, Mitsuyoshi Kitada (Department of Obstetrics and Gynecology, Kansai Medical University); Yorihiko Horikoshi (Department of Obstetrics and Gynecology, Kansai Medical University Kori); Osamu Ishiko, Yuichiro Nakai, Junko Nishio, Seiichi Yamamasu (Department of Obstetrics and Gynecology, Osaka City University Graduate School of Medicine); Jinsuke Yasuda (Department of Obstetrics and Gynecology, Matsushita Memorial Hospital); Seigo Kawai (Department of Obstetrics and Gynecology, Hoshigaoka Koseinenkin Hospital); Kazumi Yanagihara (Yanagihara Clinic); Koji Wakuda (Department of Obstetrics and Gynecology, Fujimoto Hospital); Tokio Kawashima (Kyohritsu Women's Clinic); Katsuhiko Narimoto (Ishida Hospital Obstetrics, Gynecology); Yoshihiko Iwasa (Iwasa Women's Clinic); Katsuhiko Orino (Orino Lady's Clinic); Itsuo Tsunetoh (Tsunetoh Obstetrics and Gynecology); Junichi Yoshida (Yoshida Clinic); Junichi Iito (Iito Obstetrics and Gynecology Clinic); Takuzi Kaneko (Kaneko Sanfujinka); Takao Kamiya (Kamiya Ladies Clinic); Hiroyuki Kuribayashi (Kuribayashi Clinic); Takeshi Taniguchi (Taniguchi Hospital); Hideo Takemura (Kosaka Women's Hospital); Yasuhiko Morimoto (Aizenbashi Hospital); Ichiro Matsunaga, Hajime Oda (Osaka Prefectural Institute of Public Health); Yukihiro Ohya (Division of Allergy, Department of Medical Specialties, National Center for Child Health and Development). 